\title{
Mitotic drivers-inhibitors of the Aurora B Kinase
}

\author{
Nicholas Keen • Stephen Taylor
}

Published online: 3 February 2009

(C) The Author(s) 2009. This article is published with open access at Springerlink.com

\begin{abstract}
In this article we review the basis for current anti-mitotic, anti-cancer, therapy and the potential for Aurora B kinase inhibitors as a new differentiated class of agents- "mitotic drivers". We review the current understanding of Aurora B inhibition from basic cell biology to inhibitors currently undergoing clinical trials.
\end{abstract}

Keywords Aurora Kinase · Mitosis · Anti-mitotic ·

Cell-cycle $\cdot$ Cancer Therapeutic

\section{Introduction}

Errors in mitosis can lead to genomic instability and the development of cancer. In the past decade there has been a dramatic increase in the understanding of the underlying biological machinery "that performs this essential cellular process, and the subtle ways in which it can malfunction in cancer. As a direct consequence of this increased understanding new drug targets have been identified that hold the promise of enhanced efficacy and reduced toxicity when compared to other cytotoxic approaches. One prototypical example is the Aurora B. This kinase was first demonstrated to be cancer associated (overexpressed in colon tumours as compared to adjacent normal tissue) in 1998 [1]. In the intervening period the Aurora kinases have been the

\footnotetext{
N. Keen $(\bowtie)$

AstraZeneca Pharmaceuticals, R\&D Boston,

Waltham, MA 02451, USA

e-mail: nick.keen@astrazeneca.com

S. Taylor

Faculty of Life Sciences, University of Manchester,

Michael Smith Building, Oxford Road,

Manchester M13 9PT, UK

e-mail: stephen.taylor@manchester.ac.uk
}

focus of intense academic and industrial effort currently culminating in multiple clinical trails with a diverse array of inhibitors. Here we review the background biology, current status and future directions for these exciting new anticancer agents.

\section{Antimitotics - drugs which block mitosis}

2.1 Microtubule toxins and the history of the antimitotic concept

The medicinal properties of the so called antimitotic agents has been appreciated for a long time; plant extracts containing colchicine have been used to treat gout for centuries [2]. Today, a large array of antimitotics including the taxanes (e.g. paclitaxel, docetaxel) and the vinca alkaloids (e.g. vincristine, vinblastine) are used routinely in the clinic, primarily to treat cancer [3]. In the United Kingdom, about $75 \%$ of women with ovarian cancer receive paclitaxel in combination with a DNA damaging agent as a first-line therapy [4]. The widespread use of antimitotics is well founded; two comprehensive reviews of Phase III clinical trials showed that the inclusion of taxanes in adjuvant chemotherapy regimens for breast cancer improved disease free survival and overall survival $[5,6]$.

The common feature of the antimitotics is that they all bind tubulin [3]; indeed, tubulin was discovered via its ability to bind colchicine [7]. By binding tubulin, the antimitotic compounds suppress microtubule dynamics which has a profound effect on cells during mitosis. Dynamic microtubules are required to build the bipolar spindle which is then used to accurately segregate the duplicated chromosomes to daughter cells [3]. When spindle assembly is inhibited, the spindle checkpoint is activated, thereby inducing mitotic arrest, a phenomenon 
which has been appreciated for over 100 years [8]. Indeed, its is the prolonged mitotic arrest that gives this class of compounds its name. Following prolonged mitotic arrest, tumour cells typically die, either directly in mitosis or following mitotic exit [9].

The therapeutic strength of the antimitotics is also their weakness. Dividing tumour cells need dynamic microtubules to divide, explaining why the antimitotics are effective against cancer cells. However, because microtubules are required for a plethora of cellular functions, the anti-tubulin drugs are toxic to normal cells, both dividing and nondividing, i.e. these drugs are cytotoxins. As is typical with other cytotoxins, toxicity towards normal dividing cells manifests as myelosuppression. The impact on the immune system is reversible and therefore clinically manageable. However, side affects associated with antimitotics that are not typical of other cytotoxins are peripheral neuropathies, caused by the inhibition of microtubule-dependent processes in axons and glial cells [10]. These neuropathies can manifest as loss of sensation in the extremities and can be irreversible. In the last 10 years, much efforts has been spent on trying to bypass this latter problem. If one assumes that the efficacy of the anti-tubulin drugs is due to their antimitotic properties, then in principle, compounds that prevent mitotic spindle assembly without affecting microtubules in non-dividing cells should retain anti-tumour activity but not the associated neuropathies.

\subsection{KSP inhibitors-bringing the antimitotic concept into the 21 st centaury}

The first clue that it may be possible to develop drugs which inhibit mitotic spindle assembly without affecting microtubule dynamics came from a pioneering high throughput screen using a cell-based phenotypic assay [11]. The screen was designed to identify compounds which induced mitotic arrest, just as the microtubule toxins do. However, secondary screens were then used to eliminate compounds which inhibited interphase microtubule dynamics. The screen yielded a compound which inhibited Eg5, a member of the kinesin-5 family of motor proteins. Eg5, also known as kinesin spindle protein (KSP), is a plus-end directed motor which acts as a homotetramer to cross-link and slide anti-parallel microtubules at the onset of mitosis, thereby pushing apart the centrosomes to form the bipolar spindle [12]. In the absence of $\mathrm{Eg} 5$ function, the centrosomes remain tightly clustered yielding a monopolar spindle; consequently, the inhibitor was christened Monastrol [11]. Like the tubulin agents, this spindle defect activates the spindle checkpoint resulting in mitotic arrest.

Monastrol has turned out to be a fantastic research tool, allowing cell biologists to probe the molecular mechanisms that regulate spindle assembly and mitotic progression [see e.g. [13]]. In addition, Monastrol validated KSP as an attractive anti-cancer drug target. Not only did Monastrol show that KSP was druggable, but because KSP is only expressed in proliferating cells and is required only during mitosis, selective KSP inhibitors should exert antiproliferative effects typical of the traditional antimitotics, but not the associated neuronal toxicity. Indeed, a number of KSP inhibitors have now been developed as anti-cancer drugs and clinical trials are underway [14].

One of the forerunners is Ispinesib, also known as SB715992 or CK0106023, a quinazolinone derivative which inhibits the ATPase activity of KSP with a Ki of $12 \mathrm{nM}$ [15]. Tumour cells treated in culture with submicromolar doses of Ispinesib arrest in mitosis with monopolar spindles and then undergo apoptosis. This leads to growth inhibition in a variety of tumour lines, with a mean $\mathrm{GI}_{50}$ value of $364 \mathrm{nM}$. Ispinesib appears to be selective for KSP and the mode of action is due to reduced ADP release. In mousebased xenograft models, Ispinesib inhibits tumour growth, and encouragingly, monopolar spindles were evident in tumour biopsies, indicating that KSP was targeted in vivo. Importantly, in Phase I clinical trials with Ispinesib and two other KSP inhibitors, namely SB-743921 and MK-0731, neuropathy was not reported as a major side effect; as expected for a cytotoxin, the dose limiting toxicities were neutropenia [16-18]. Thus, the KSP inhibitors appear to have achieved one major goal, namely the elimination of neurotoxicities. Clearly however, this will count for little if they do not exert anti-tumour effects comparable with the existing anti-tubulin agents. Thus far, only a handful of Phase II trials have reported. Although no objective responses were observed, it is important to note that these trials focussed on patients with recurrent or metastatic diseases including hepatocellular carcinoma, squamous cell carcinoma and malignant melanoma. A key issue for the future therefore will be to define which tumour types are most likely to respond to KSP inhibitors.

\subsection{Extending the antimitotic concept—other agents that inhibit spindle assembly}

KSP is only one of many proteins required for spindle assembly. In the last 10-15 years our understanding of the molecular mechanisms responsible for spindle assembly, chromosome alignment, the control of mitotic progression and cytokinesis has expanded enormously. This basic knowledge has therefore presented us with a plethora of novel anti-cancer targets; in principle, inhibition of any protein required for spindle assembly may yield antimitotic phenotypes typical of the microtubule toxins and the KSP inhibitors. The list of potential candidates is now expanding even faster as genome wide RNAi screens identify yet more molecules required for spindle assembly [19-21]. However, 
not every protein lends itself to therapeutic intervention; rather, only a subset of proteins are amenable to drug discovery, leading to the concept of "the druggable genome" [22]. One of the largest families in the druggable genome is the protein kinase family, comprising of both serine/threonine and tyrosine kinases. Importantly, several protein kinases are required for spindle assembly, including the Aurora and Polo-like kinases [23]. Not surprisingly therefore, based on the antimitotic paradigm, these protein kinases have received much attention as potential anticancer drug targets [24].

\subsection{Aurora A inhibitors-another route to monopolar spindles}

The Aurora family of protein kinases is conserved from yeast to man [for recent reviews see [25-27]]. While budding and fission yeast express a single Aurora kinase, Ipl1 and Ark1 respectively, higher eukaryotes express at least two Aurora kinases, A and B, with mammals expressing a third, Aurora C. The founder member of the family, Drosophila Aurora A, was identified because Aurora mutations caused abnormal mitoses, largely due to a failure in centrosome separation yielding monopolar spindles [28]. Based on the antimitotic concept outlined above, this observation alone suggests that Aurora A might be an attractive anti-cancer drug target. Indeed, Aurora A has received an enormous amount of attention from both the academic and pharmaceutical industry. However, this interest stems largely from observations that accompanied the discovery of the human homologue [1]. Firstly, human Aurora A was discovered in a screen to identify protein kinases overexpressed in colon cancer. Secondly, Aurora A localises to $20 \mathrm{q} 13$, a region of the genome that is amplified in some tumours and is associated with poor prognosis. And finally, overexpression of wild type Aurora A - but not a catalytically inactive mutant - transformed rodent cells in vitro. The original rationale behind developing Aurora A inhibitors therefore was not based on the antimitotic concept described above, but rather on the principle that Aurora A was an oncogene. Thus, the rationale for targeting Aurora A was that it inhibition might restrain cellular proliferation in much the same way that Imatinib inhibits cells expressing BCR-Abl [29].

A plethora of Aurora inhibitors have now been described [24]. The majority of the early inhibitors yielded phenotypes consistent with inhibition of Aurora B (see below) raising the question as to whether it was possible to inhibit Aurora A in cells with a small molecule. However, in 2006 it was it shown using a dual Aurora A/B inhibitor, ZM3 that it was possible to suppress Aurora A kinase activity in cells with a small molecule [30]. Encouragingly, this resulted in a monopolar spindle phenotype, confirming the observations derived from model organisms [25]. Subsequently, a selective Aurora A inhibitor has been described, namely MLN8054. Developed by Millennium Pharmaceuticals, MLN8054 has a benzazepine core scaffold with a fused aminopyrimidine ring and represents the first Aurora inhibitor with substantial selectivity for Aurora A over B having in vitro $\mathrm{IC}_{50}$ values of 4 and $172 \mathrm{nM}$, respectively [31]. In cell culture assays MLN8054 also shows selectivity for Aurora A over B. At $1 \mu \mathrm{M}$, MLN8054 blocks Aurora A T-loop phosphorylation on T288, but little effect on Histone H3 serine 10 phosphorylation is observed, indicating little effect on Aurora B activity. Consistent with established roles for Aurora A, MLN8054 induces abnormal spindles, often with unseparated centrosomes, and delays progression through mitosis [31]. This is accompanied by reduced phosphorylation and localization of the Aurora A substrate TACC3 [32]. These observations are entirely consistent with data from model systems, indicating that MLN8054 has potential, not only as a new chemical-biology tool for probing Aurora A function, but also as a new antimitotic agent. Surprisingly however, in contrast to the terminal mitotic arrest induced by anti-microtubule agents and KSP inhibitors, MLN8054-treated cells ultimately assemble bipolar spindles, possibly via the formation of ectopic poles, and then divide, albeit with segregation errors [33]. Whether this is because of incomplete Aurora A inhibition or the existence of Aurora-A-independent spindle assembly pathways remains to be seen. Despite the ability of MLM8054-treated cells to eventually divide, MLN8054 does exert anti-tumour effects in mouse xenograft models. While the exact mechanism remains to be clarified, the antitumour effects are accompanied by inhibition of Aurora A activity in vivo as well as spindle defects, consistent with the notion that the phenomenon is mediated via inhibition Aurora A.

\subsection{Plk1 inhibitors-more monopolar spindles}

The founder member of the Polo-like-kinase (Plk) family was also identified in Drosophila, with polo mutants displaying a penetrant spindle assembly defect [34, 35]. Mammals express four Plks, with Plk1 being the most understood. Plk1 localizes to centrosomes, kinetochores, and then the central spindle in anaphase [Recently reviewed in $[5,36]]$. Plk1's functions are not simply restricted to spindle assembly; it has been implicated in the activation of Cdk1-cyclin B at mitotic entry; centrosome maturation and spindle assembly; the release of cohesin from chromosome arms in prophase; and the activation of the $\mathrm{APC} / \mathrm{C}$ by direct phosphorylation and inhibition of Emi1. Plk1 also triggers the initiation of cytokinesis by recruiting Ect2, an exchange factor for the RhoA GTPase, to the central spindle in anaphase [Reviewed in [24]]. Despite being involved in a 
several mitotic processes, the predominant phenotype observed following Plk1 inhibition is a spindle assembly defect leading to prolonged mitotic arrest. Thus, in contrast to Aurora A inhibitors, a Plk1 inhibitor may be more reminiscent of the phenotypes induced by anti-microtubule agents and KSP inhibitors.

In the last few years a number of Plk1 inhibitors have been described. Plk1 is inhibited by several generic kinase inhibitors such as staurosporine and wortmannin [Reviewed in [24]]. However, the low specificity of these compounds has limited their use as Plk1 inhibitors. The compound ON01910 was reported to be a Plk1 inhibitor [37], but in subsequent studies no inhibition of purified Plk1 was observed up to $30 \mu \mathrm{M}$ [38]. Furthermore, the cellular phenotypes induced by ON01910 were not characteristic for Plk1 inactivation $[39,40]$. More selective Plk1 inhibitors have recently become available, [Reviewed in [24]]. Of particular note are BI 2536 and TAL. BI 2536, developed by Boehringer Ingelheim, is a dihydropteridinone that potently inhibits Plk1 in vitro with an $\mathrm{IC}_{50}$ of $1 \mathrm{nM}$. Phenotypes induced by BI 2536 are consistent with Plk1 inhibition indicating that it is a good tool for probing Plk1 function [38, 39]. TAL is a thiazolidinone developed by Bayer Schering Pharma that inhibits Plk1 in vitro with an $\mathrm{IC}_{50}$ of $19 \mathrm{nM}$. Again, the phenotypes observed in cell based assays are entirely consistent with Plk1 inhibition [41]. Importantly, both BI 2536 and TAL induce a penetrant monopolar spindle phenotype which in turn activates the spindle checkpoint resulting in a mitotic arrest phenotype. Thus, in contrast to Aurora A inhibitors, Plk1 inhibitors do indeed yield phenotypes more typical of those induced by anti-microtubule agents and KSP inhibitors. Encouragingly, BI 2536 exerts anti-tumour effects in xenograft models [38]. When nude mice harbouring HCT 116 colon cancer derived tumours were infused i.v. with consecutive cycles of BI 2536 twice per week, complete tumor suppression was observed. Importantly, $24 \mathrm{~h}$ post-infusion, a massive accumulation of mitotic cells was observed which was then followed $24 \mathrm{~h}$ later by a wave of apoptosis. These observations indicate that in vivo, Plk1 inhibition does indeed block mitotic progression which in turn results in cell death. A number of Plk1 inhibitors are now undergoing clinical evaluation.

\section{A new concept-mitotic drivers}

\subsection{Inhibition of Aurora B overrides the spindle checkpoint}

As mentioned above, the initial Aurora inhibitors described, such as ZM447439 and Hesperadin, were relative selective for Aurora B over A [30, 42-44]. Even dual Aurora inhibitors, such as VX-680, yielded phenotypes more consistent with Aurora B inhibition [45]. In contrast to Aurora A, Aurora B is not required for spindle assembly. Rather, along with survivin, INCENP, and Borealin, Aurora B is a component of the chromosome passenger complex (CPC) which localizes to centromeres in prometaphase [Reviewed in [27]]. It then relocates to the spindle midzone following anaphase onset. Prior to anaphase, Aurora B Promotes kinetochore biorientation by regulating kinetochore-microtubule interactions. Aurora B is also required for spindle checkpoint activation and cytokinesis. In prophase, Aurora B phosphorylates histone H3 on serines 10 and 28. When Aurora B is inhibited, cells fail to biorient their chromosomes. Normally, this would be anticipated to activate the spindle checkpoint leading to a mitotic arrest. However, because Aurora B is also required for spindle checkpoint function, Aurora-Bdeficient cells enter anaphase and exit mitosis despite the presence of chromosome malorientations. Cytokinesis then also fails yielding a cell in G1 but with a 4n DNA content (see below for more details). This phenotype is clearly different from that exerted by the so called antimitotic drugs; where as the microtubule toxins, inhibitors of KSP, Aurora A and Plk1 all activate the spindle checkpoint causing cells to arrest in mitosis, inhibition of Aurora B overrides the checkpoint and drives cells through an aberrant mitosis. Therefore, it is not appropriate to consider Aurora B inhibitors as antimitotic agents; rather, we suggest that Aurora B inhibitors be termed "mitotic drivers".

\section{Inhibitors of the Aurora B kinase}

\subsection{Lessons from cell culture}

Since the original association between Aurora A and B kinase overexpression and cancer was identified, there has been a huge effort from both academic and industrial groups to develop small molecule kinase inhibitors to these targets. The first Aurora inhibitiors to be described were ZM447439, Hesperadin and VX680[42, 43, 45] and these remain the best characterised in terms of the publically available literature [Reviewed in [24]]. ZM447439 shows 20 -fold selectivity for Aurora B over A and has proved to be a useful tool for probing Aurora B function. Similarly, Hesperadin has been used extensively to probe Aurora B function; note however that the cytokinesis failure induced by Hesperadin may be due to an off-target effect in addition to the inhibition of Aurora B [30]. VX680 is a dual A/B inhibitor but as with the majority of Aurora inhibitors - the exception being MLN8054 - the predominant phenotypes in cell based assays arise due to Aurora B inhibition yielding the mitotic driver phenotype described above. This, along with the roles of Aurora B in cytokinesis, leads 
to a highly abnormal mitosis with a failure of cytokinesis but without a cell cycle arrest. These cells maintain an apparently normal coordinated expression of key cell cycle regulators such as the cyclins, and will undergo additional rounds of S-phase and failed mitosis in the presence of an inhibitor (endeoreduplication) producing enlarged, polyploid cells with multiple centrosomes. When the drug is removed, it is likely that these cells attempt mitosis but without a bipolar spindle and in a highly uncoordinated manner, literally "tearing" the genome apart and leading to cell death. When tested in vitro, all of the reported Aurora B inhibitors are highly effective at killing tumour cells in vitro although the exact mechanism is unclear. Importantly, since Aurora B is only active in mitosis, inhibitors should have no effect on cells that do not pass through mitosis. Indeed, MCF7 cells retained their cloning potential when exposed to ZM447439 while arrested in G1 [42].

\subsection{Lessons from animal models}

Encouragingly, those Aurora kinase inhibitors which are compatible with in vivo dosing show excellent anti-tumour activity against human tumour cell lines grown as xenografts in rodents. VX-680 caused a marked reduction in tumour size in a human promyelocytic leukemia (HL-60) xenograft model [45]. In nude mice treated with VX-680 at $75 \mathrm{mg} / \mathrm{kg}$, twice a day intraperitoneally for 13 days, mean tumour volumes were reduced by $98 \%$. The authors reported that VX-680 was well tolerated, with a small decrease in body weight observed only at the highest dose (5\% decrease at $75 \mathrm{mg} / \mathrm{kg}$ b.i.d). VX-680 also induced tumour regression in pancreatic and colon xenograft models. Encouragingly, inhibition of phosphorylation of histone $\mathrm{H} 3$ and an increase in apoptosis were observed in histological sections of tumours from treated animals indicating that Aurora B was effectively inhibited at the doses used, and that this killed tumour cells in vivo.

Another Aurora kinase inhibitor, PHA-739358 [46], was also very active in vivo - in this case the authors extended the work beyond classic tumour xenografts to perhaps more relevant spontaneous tumour models and transgenic tumour models. When PHA-739358 was evaluated classic nude mouse xenograft models, significant growth inhibition was reported (up to $98 \%$ in HL60 xenografts dosed with $60 \mathrm{mg}$ / $\mathrm{kg}$ /day PHA-739358 i.v. for 5 days). Toxicity was reported to be limited with minimal body weight loss and mild myelosuppression, both of which were transient with recovery after treatment. The authors also utilised a rat DMBA-induced primary mammary carcinoma model that displays many similarities with human breast cancer. Administration of $25 \mathrm{mg} / \mathrm{kg}$ PHA-739358 to these rats (twice a day, i.v.) resulted in $75 \%$ inhibition of tumour growth with complete regression in one animal. The efficacy was similar to that achieved in the xenograft mouse models. Finally, the authors evaluated the efficacy of PHA-739358 in the TRAMP transgenic mouse prostate cancer model. When TRAMP mice were treated for 5 days bd i.v. with $30 \mathrm{mg} / \mathrm{kg}$ of PHA-739358, 3 out of 16 mice showed tumor regression up to $80 \%$. Again the authors reported suppression of phospho histone $\mathrm{H} 3$ and elevation of apoptotic markers in treated tumours.

More recent work [47] examined the sequence of events in tumours treated with AZD1152 (a selective Aurora B inhibitor - see Table 1) and provides a clear insight into the fate in vivo of tumour cells exposed to Aurora B inhibitors. Infusion of AZD1152 to a human tumour xenograft bearing animal induces a cascade of events - suppression of histone H3 phosphorylation, progression of mitosis without biorentied chromosomes, failure of cytokinesis leading to enlarged polypolid cells and elevated levels of apopotosis and necrosis in the tumour. The authors also evaluated the effect of this Aurora kinase inhibitor on normal bone marrow in the treated animals. As would be expected, inhibition of Aurora B led to loss of bone marrow and concomitant neutrapaenia. Perhaps surprisingly, there was little evidence for enlarged or multinucleated cells in treated bone marrow suggesting that the fate of "normal" bone marrow cells exposed to an Aurora B inhibitor may be different than tumour cells (immediate apoptosis in response to accelerated mitotic slippage?). Encouragingly, the bone marrow in treated animals recovered to apparently normal levels 4 days post treatment whilst the effects on the tumour were prolonged-suggesting that a therapeutic index may be obtainable for these agents.

\subsection{Clinical development}

There are currently more multiple Aurora kinase inhibitiors in clinical trials. With the exception of MLN8054 the majority are Aurora B selective or dual A/B inhibitors (Table 1). At present there is little publicly available information and therefore we will summarize what is know about the most advanced compounds. In a phase II trial, PH739358 from Nerviano Life Sciences has resulted in seven reported cases of stable disease in the solid tumour setting (out of 36 patients dosed; 4 for $>7$ months) and partial/complete responses in the haematological area (CML). PH739358 is administered as a 6-hour infusion weekly (×3 q28d). Disappointingly, MK0457/ VX680 (Merck/Vertex) has been discontinued in phase II despite responses again in CML. This agent was halted due to reported issues with QTc prolongation. AZD1152, which is selective for Aurora B is in Phase I in both solid and haematological (AML) tumours, although there has been no publication of activity to date. Dose limiting toxicology 


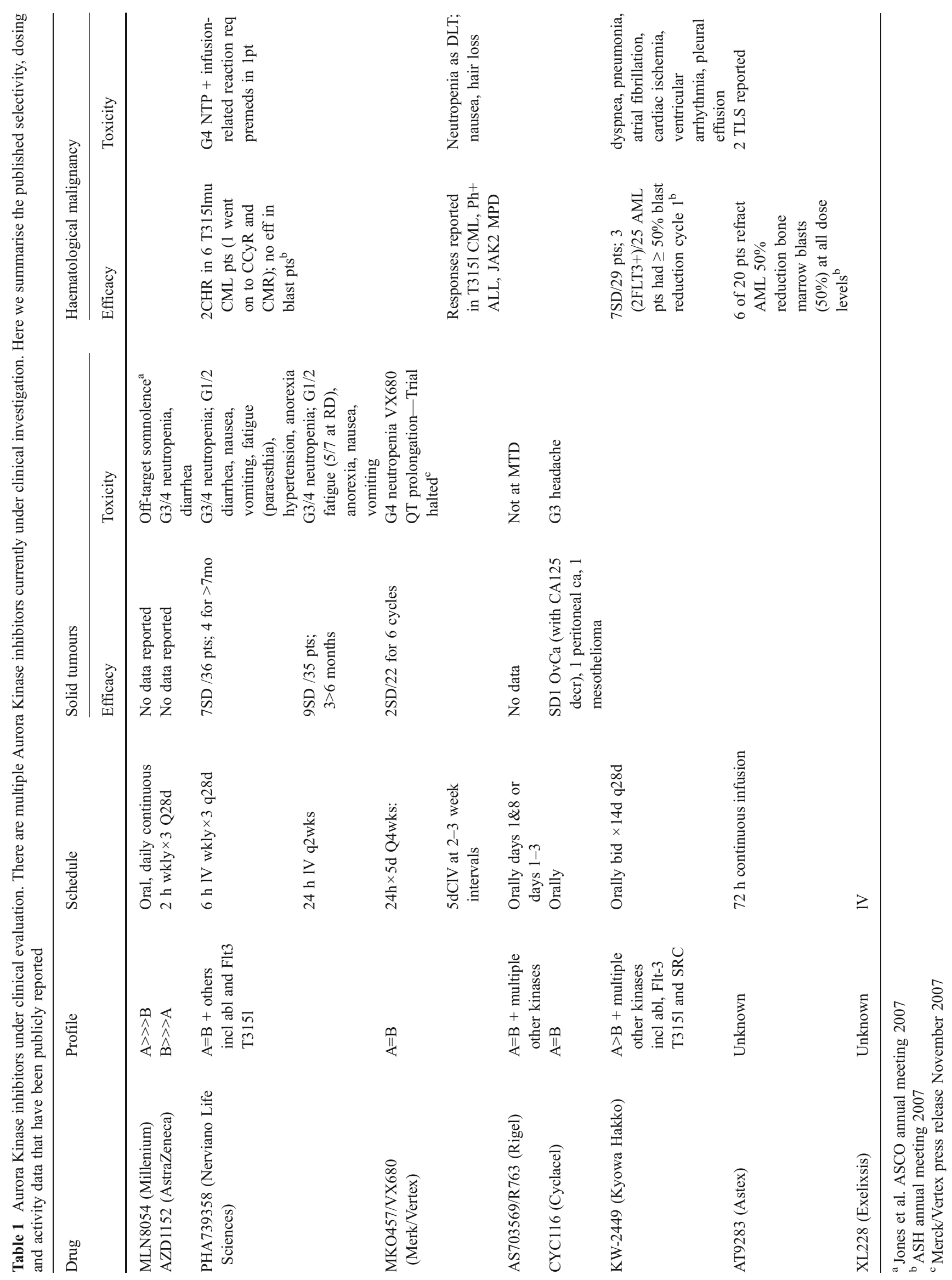


reported for the majority of these agents seems fairly similar to the "classic" anti-mitotic cytotoxics-bone marrow suppression (neutropenia), alopecia, nausea and vomiting and diarrhoea being typical. At this stage we cannot determine if the therapeutic index for Aurora kinase inhibitors is greater, less than or equal to classic anti-mitotic cytotoxics. Nevertheless, it is very encouraging that responses are being observed (principally in the haematological area) so early in the development of this class of new drugs.

\section{Clinical challenges-how can we increase the chance of beneficial outcomes?}

We perceive that there are three critical biological questions that will need to be addressed in order to most effectively develop these agents in the clinic.

5.1 How does one know that a biologically effective dose has been achieved in the tumour?

This should be easy to answer in theory-suppression (or elevation in the case of an Aurora A inhibitor) of phosphorylation of histone $\mathrm{H} 3$ can be readily measured, for example by using immunohistochemical techniques. For Aurora B inhibitors this provides a direct measure of target inhibition at the site, an ideal "biomarker". Alternatively, more common markers of proliferation and apoptosis (e.g. Ki67, cleaved caspases etc) could be employed. Another, although perhaps less desirable, alternative is to rely on measuring suppression of bone marrow as a clinical surrogate for biological activity of these agents.

5.2 Which tumour type(s) either from tissue of origin or based on genetic defects will be most likely to respond?

This is a much more complex issue. It is clear that response does not relate simply to expression levels of these kinases, apart from at the crudest level — cells must be passing through mitosis where the kinases are expressed and active in order for the drugs to work, and tissues with a high mitotic index by definition will express high levels of Aurora kinases and perhaps be more likely to respond to treatment. Perhaps unsurprisingly, it is clear that many of the current clinical agents are being tested in haematological cancers that have a combination of a high mitotic index and ease of accessibility to tumour tissue for biomarker measurement (indeed these agents seem very active in in vitro and in vivo models for haematological disease as outlined above for VX-680 and PHA-739358). However, we do not currently understand what other factors, genetic or environmental, contribute to the response (G1 arrest, apoptosis, continued cycling, senescence etc) of tumour cells to Aurora B inhibition. Indeed, the response of even apparently clonogenic cell lines to "simple" antimitotics can be heterogenous and extremely complex [9]. This is an area that deserves significant research, since if defined genetic lesions do confer sensitivity to Aurora kinase inhibition this would have direct medical benefit—clinical trials could be greatly reduced in size, increased in speed and become genuinely hypothesis driven.

5.3 What duration of inhibition is necessary for obtaining an optimal therapeutic index?

This is a seemingly trivial question, but in real life very complex to address. The critical issue here is to reduce effects on normal tissue but optimise effects on tumour. As we outlined above, the tumour cells will need to pass through mitosis in the presence of the drug in order to be affected. This typically sets a minimum exposure time based on the proliferative index of the tumour. The maximum is a function of how much ablation of normal proliferating cells can be tolerated. For most of the current agents in the clinic the minimum appears to be of the order of a few hours out to a maximum of 7 days continual infusion, with a wide variety of cycles and schedules being evaluated. Again, it cannot be emphasised strongly enough how useful it would be to be able to pre-select responsive tumours based on their genotype or other characteristics in order to rapidly narrow down these dosing regimens.

\section{The future}

Within a short space of time the initial observations that the Aurora kinases are overexpressed in cancer have been translated into the development of drugs that have suitable properties to investigate their role as a potential new class of anti-cancer agents, the "mitotic drivers". Many clinical trials are now underway with a spectrum of agents that cover the full range of kinase selectivity from broadly targeting to exquisite selectivity for a given Aurora kinase. So far, the data emerging from the clinic are both encouraging (responses) and disappointing (toxicology). What directions could future research take in order to enable us to better use the current generation of Aurora inhibitors - and are there additional opportunities to target the machinery of mitosis that may yield genuinely tumour cell selective killing?

6.1 How can we predict responsive tumour populations?

As we outlined above we believe that it would provide a major benefit to patients if the genetic basis of the outcome 
of exposure to Aurora kinase inhibitors could be understood. This is a very significant problem in oncology drug discovery in general, and even in the case of apparently "simple" agents such as the tubulin binding anti-mitotics can induce different fates in different cells within an apparently clonal population. Adding in the complexity of tumour cell heterogeneity and response to microenvironment and this becomes a very challenging task.

There are however ways that this could be approachedfor example genome scale siRNA "synthetic lethal screen" in the presence of an Aurora kinase inhibitor (analogous those performed for PARP inhibitors [48]) that may reveal genes that interact with Aurora B in a lethal manner. If loss of any of these genes (or pathways in which these genes operate) occurs in specific tumour populations then this could potentially identify tumours that are more sensitive to inhibition than normal tissue. There are of course many other approaches that could be taken. Another area that is currently not well understood is the behaviour of Aurora kinase inhibition in combination with other anti-cancer therapeutics from classic cytotoxics through to more recent specific inhibitors of signal transduction. Identifying combinations that have synergistic effects in tumours is clearly a priority.

\subsection{Drug-resistance?}

In common with the emergence of resistance seen with other kinase inhibitors in the oncology setting, it is likely that the selective pressure placed upon the tumour, coupled with the inherent genomic instability of tumour cells will select for evolution of inhibitor resistant kinase mutants. Indeed, this has already been demonstrated in the in vitro setting. A recent screen showed that HCT116 cells can become resistant to ZM447439 by acquiring mutations in Aurora B [30]. Two mutations mapped to the active site and probably occlude inhibitor binding while a third mutation, near the activation loop, appears to hyperactivate the kinase. These mutations also rendered Aurora B resistant to other inhibitors namely VX-680, Hesperadin and MLN8054. It would therefore be valuable to generate and characterise the behaviour of resistant mutants to the current agents in the clinic. This could lead to the development of new compounds that will either overcome resistance mechanisms or be intrinsically more difficult to evolve resistance to (for example by allosteric rather than ATP competitive inhibition).

\subsection{Can the mitotic driver concept identify other targets?}

Distinguishing antimitotics from mitotic drivers is not a question of semantics. At present, it is not clear how antimitotic agents actually exert their anti-tumour effects. It is well established that antimitotic compounds activate the spindle assembly checkpoint (SAC) leading to mitotic arrest. Following prolonged arrest, a number of outcomes are possible (Fig. 1). While some cells die in mitosis, others exit mitosis without dividing and return to interphase. Once back in interphase, some lines undergo cell-cycle arrest, others die, and others rereplicate their genomes, i.e., endocycle [9]. What is not clear however is which of these phenotypes is the most desirable in vivo. Indeed, in only a
Fig. 1 The mitotic driver concept. Traditional antimitotic agents such as the microtubule toxins disrupt spindle assembly which leads to a mitotic arrest phenotype. By contrast, mitotic drivers - the prototypes being inhibitors of the Aurora B kinase-drive cells out of an aberrant mitosis

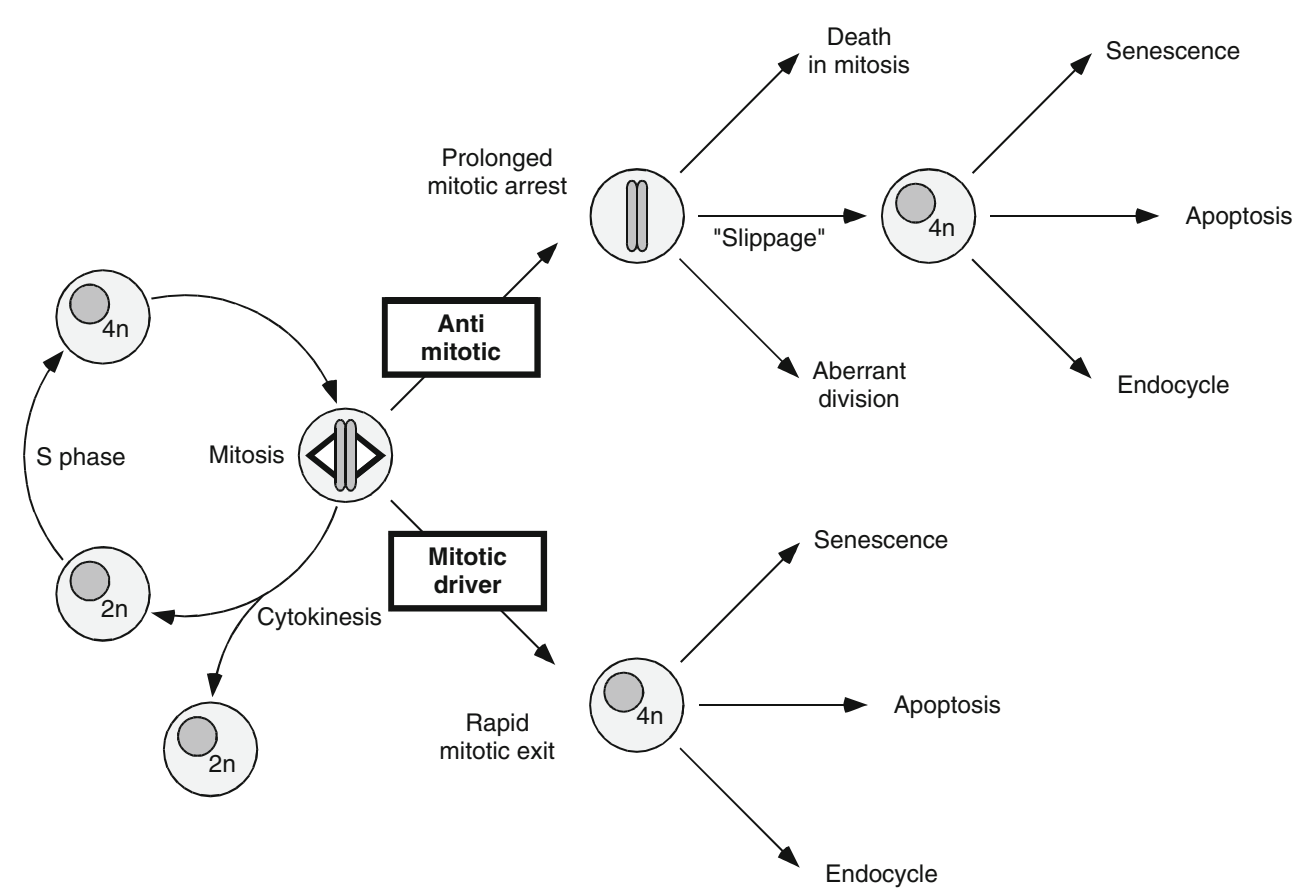


few cell lines is the death in mitosis the dominant behavior. Most cells eventually return to interphase, a process known as slippage, before eventually undergoing apoptosis. Perhaps slippage and death in interphase, as opposed to death in mitosis, is the more clinically desirable outcome. If this is the case, then other mitotic drivers, which in effect accelerate slippage causing exit from an aberrant mitosis, may also have merit as an anti-cancer agent. In turn, if this is the case, it opens up the possibility of exploring a number of other targets, in particular other kinases which are required for spindle checkpoint function. A number of kinases are required for checkpoint function in humans including Bub1, BubR1, Mps1, Tao1 and Prp4 [49]. Inhibitors of these kinases, either alone or in combination with antimitotic agent would be expected to drive cells out of an aberrant mitosis, in other words to act as mitotic drivers.

\subsection{Can we inhibit mitosis selectively in tumour cells?}

Like the antimitotics and Aurora B inhibitors, SAC inhibitors would affect all dividing cells and would therefore be expected to be cytotoxins. The preference would be to identify targets that preferentially affect tumour cells over normal cells. One way to do this was reported very recently in an elegant paper by Kwon and colleagues [20]. They utilised the observation that tumour cells frequently carry multiple centrosomes but still form bipolar spindles. They performed a genetic screen to identify genes that were responsible for centrosome clustering in Drosophila cells carrying $>2$ centrosomes and then characterised human homologues of these genes in tumour and normal cells. siRNA inhibition of one of these genes, HSET, led to unclustering of centrosomes, multipolar mitosis and cell death specifically in tumour cells with $>2$ centrosomes, whilst no effect was observed in normal cells or tumour cells with a normal centrosome complement. This suggests that inhibition of HSET, which is an (-) end directed kinesin motor protein (and therefore likely to be amenable to small molecule inhibition) could lead to tumour cell specific killing. Furthermore, it should be relatively straightforward to identify tumour populations with $>2$ centrosomes, greatly focussing clinical trials, should inhibitors be developed.

There are clearly other opportunities to identify and prey upon tumour cell specific abnormalities in mitosis that could lead to tumour specific killing. This will be an exciting area for future research.

\section{Summary}

In the 10 years since the initial association between Aurora kinase overexpression and cancer was identified huge progress has been made in both understanding the basic biological role of these kinases and in developing small molecule inhibitors with the rare combination of properties that enables them to be dosed to man. Elegant work using these agents and genetic tools has revealed a unique mechanism of action-forced mitotic drive rather than a mitotic arrest. This seems very effective in killing tumour cells in model systems. Encouragingly there are also indications of activity against real human tumours emerging. This is impressive given that the majority of patients in these trials will have failed pre-existing therapies, including anti-mitotics and indicates that Aurora kinase inhibitors may have clinical utility.

However there still remains much work to be done to understand how these agents can be most effectively used to benefit patients with cancer, and to better understand the mechanisms by which interference with basic mitotic mechanisms can kill tumour cells.

Improved understanding here will yield direct benefit to patients, and may yield the "holy grail"-drugs which combine the lethality of cytotoxic agents with specificity for tumour cells.

Acknowledgements SST is a Cancer Research UK Senior Fellow

Competing Financial Interests SST-none.

NK is a full time employee of AstraZeneca Pharmaceuticals LLC.

Open Access This article is distributed under the terms of the Creative Commons Attribution Noncommercial License which permits any noncommercial use, distribution, and reproduction in any medium, provided the original author(s) and source are credited.

\section{References}

1. Bischoff, J. R., Anderson, L., Zhu, Y., Mossie, K., Ng, L., Souza, B., et al. (1998). A homologue of Drosophila aurora kinase is oncogenic and amplified in human colorectal cancers. European Molecular Biology Organization journal, 17(11), 3052-3065.

2. Nuki, G., \& Simkin, P. A. (2006). A concise history of gout and hyperuricemia and their treatment. Arthritis Res Ther, 8(Suppl 1), S1.

3. Jordan, M. A., \& Wilson, L. (2004). Microtubules as a target for anticancer drugs. Nature Reviews Cancer, 4(4), 253-265.

4. National Institute for Clinical-Excellence. (2003). Guidance on the use of paclitaxel in the treatment of ovarian cancer. Technology Appraisal No. 55 http://www.nice.org.uk/guidance/ index.jsp?action $=$ byID\&o $=11486$.

5. Bria, E., Nistico, C., Cuppone, F., Carlini, P., Ciccarese, M., Milella, M., et al. (2006). Benefit of taxanes as adjuvant chemotherapy for early breast cancer: pooled analysis of 15,500 patients. Cancer, 106(11), 2337-2344.

6. Estevez, L. G., Munoz, M., Alvarez, I., Fernandez, Y., GarciaMata, J., Ruiz-Borrego, M., et al. (2007). Evidence-based use of taxanes in the adjuvant setting of breast cancer. A review of randomized phase III trials. Cancer Treat Rev.

7. Borisy, G. G., \& Taylor, E. W. (1967). The mechanism of action of colchicine. Binding of colchincine- $3 \mathrm{H}$ to cellular protein. Journal of Cell Biology, 34(2), 525-533. 
8. Mitchison, T. J., \& Salmon, E. D. (2001). Mitosis: a history of division. Nature Cell Biology, 3(1), E17-21.

9. Gascoigne, K. E., \& Taylor, S. S. (2008). Cancer cells display profound intra- and interline variation following prolonged exposure to antimitotic drugs. Cancer Cell.

10. Mielke, S., Sparreboom, A., \& Mross, K. (2006). Peripheral neuropathy: a persisting challenge in paclitaxel-based regimes. European journal of cancer, 42(1), 24-30.

11. Mayer, T. U., Kapoor, T. M., Haggarty, S. J., King, R. W., Schreiber, S. L., \& Mitchison, T. J. (1999). Small molecule inhibitor of mitotic spindle bipolarity identified in a phenotypebased screen. Science, 286(5441), 971-974.

12. Kapitein, L. C., Kwok, B. H., Weinger, J. S., Schmidt, C. F., Kapoor, T. M., \& Peterman, E. J. (2008). Microtubule crosslinking triggers the directional motility of kinesin-5. Journal of Cell Biology.

13. Kapoor, T. M., Lampson, M. A., Hergert, P., Cameron, L., Cimini, D., Salmon, E. D., et al. (2006). Chromosomes can congress to the metaphase plate before biorientation. Science, 311(5759), 388391.

14. Bergnes, G., Brejc, K., \& Belmont, L. (2005). Mitotic kinesins: prospects for antimitotic drug discovery. Curr Top Med Chem, 5(2), 127-145.

15. Sakowicz, R., Finer, J. T., Beraud, C., Crompton, A., Lewis, E., Fritsch, A., et al. (2004). Antitumor activity of a kinesin inhibitor. Cancer Research, 64(9), 3276-3280.

16. Knox, J. J., Gill, S., Synold, T. W., Biagi, J. J., Major, P., Feld, R., et al. (2008). A phase II and pharmacokinetic study of SB715992, in patients with metastatic hepatocellular carcinoma: a study of the National Cancer Institute of Canada Clinical Trials Group (NCIC CTG IND.168). Invest New Drugs, 26(3), 265-272.

17. Lee, C. W., Belanger, K., Rao, S. C., Petrella, T. M., Tozer, R. G., Wood, L., et al. (2008). A phase II study of ispinesib (SB-715992) in patients with metastatic or recurrent malignant melanoma: a National Cancer Institute of Canada Clinical Trials Group trial. Invest New Drugs, 26(3), 249-255.

18. Tang, P. A., Siu, L. L., Chen, E. X., Hotte, S. J., Chia, S., Schwarz, J. K., et al. (2008). Phase II study of ispinesib in recurrent or metastatic squamous cell carcinoma of the head and neck. Invest New Drugs, 26(3), 257-264.

19. Kittler, R., Putz, G., Pelletier, L., Poser, I., Heninger, A. K., Drechsel, D., et al. (2004). An endoribonuclease-prepared siRNA screen in human cells identifies genes essential for cell division. Nature, 432(7020), 1036-1040.

20. Kwon, M., Godinho, S. A., Chandhok, N. S., Ganem, N. J., Azioune, A., Thery, M., et al. (2008). Mechanisms to suppress multipolar divisions in cancer cells with extra centrosomes. Genes and Development, 22, 2189-2203.

21. Whitehurst, A. W., Bodemann, B. O., Cardenas, J., Ferguson, D., Girard, L., Peyton, M., et al. (2007). Synthetic lethal screen identification of chemosensitizer loci in cancer cells. Nature, 446 (7137), 815-819.

22. Hopkins, A. L., \& Groom, C. R. (2002). The druggable genome. Nat Rev Drug Discov, 1(9), 727-730.

23. Nigg, E. A. (2001). Mitotic kinases as regulators of cell division and its checkpoints. Nature reviews. Molecular cell biology, 2(1), 21-32.

24. Taylor, S., \& Peters, J. M. (2008). Polo and Aurora kinases: lessons derived from chemical biology. Current opinion in cell biology, 20(1), 77-84.

25. Barr, A. R., \& Gergely, F. (2007). Aurora-A: the maker and breaker of spindle poles. Journal of Cell Science, 120(Pt 17), 2987-2996.

26. Keen, N., \& Taylor, S. (2004). Aurora-kinase inhibitors as anticancer agents. Nature reviews. Cancer, 4(12), 927-936.
27. Ruchaud, S., Carmena, M., \& Earnshaw, W. C. (2007). Chromosomal passengers: conducting cell division. Nature reviews. Molecular cell biology, 8(10), 798-812.

28. Glover, D. M., Leibowitz, M. H., McLean, D. A., \& Parry, H. (1995). Mutations in aurora prevent centrosome separation leading to the formation of monopolar spindles. Cell, 81(1), 95-105.

29. Sherbenou, D. W., \& Druker, B. J. (2007). Applying the discovery of the Philadelphia chromosome. Journal of clinical investigation, 117(8), 2067-2074.

30. Girdler, F., Gascoigne, K. E., Eyers, P. A., Hartmuth, S., Crafter, C., Foote, K. M., et al. (2006). Validating Aurora B as an anti-cancer drug target. Journal of Cell Science, 119(Pt 17), 3664-3675.

31. Manfredi, M. G., Ecsedy, J. A., Meetze, K. A., Balani, S. K., Burenkova, O., Chen, W., et al. (2007). Antitumor activity of MLN8054, an orally active small-molecule inhibitor of Aurora A kinase. Proceedings of the National Academy of Sciences of the United States of America, 104(10), 4106-4111.

32. LeRoy, P. J., Hunter, J. J., Hoar, K. M., Burke, K. E., Shinde, V., Ruan, J., et al. (2007). Localization of human TACC3 to mitotic spindles is mediated by phosphorylation on Ser558 by Aurora A: a novel pharmacodynamic method for measuring Aurora A activity. Cancer Research, 67(11), 5362-5370.

33. Hoar, K., Chakravarty, A., Rabino, C., Wysong, D., Bowman, D., Roy, N., et al. (2007). MLN8054, a small-molecule inhibitor of Aurora A, causes spindle pole and chromosome congression defects leading to aneuploidy. Molecular and cellular biology, 27 (12), 4513-4525.

34. Llamazares, S., Moreira, A., Tavares, A., Girdham, C., Spruce, B. A., Gonzalez, C., et al. (1991). polo encodes a protein kinase homolog required for mitosis in Drosophila. Genes and development, 5(12A), 2153-2165.

35. Sunkel, C. E., \& Glover, D. M. (1988). polo, a mitotic mutant of Drosophila displaying abnormal spindle poles. Journal of Cell Science, 89(Pt 1), 25-38.

36. Strebhardt, K., \& Ullrich, A. (2006). Targeting polo-like kinase 1 for cancer therapy. Nature reviews. Cancer, 6(4), 321-330.

37. Gumireddy, K., Reddy, M. V., Cosenza, S. C., Boominathan, R., Baker, S. J., Papathi, N., et al. (2005). ON01910, a non-ATPcompetitive small molecule inhibitor of Plk1, is a potent anticancer agent. Cancer Cell, 7(3), 275-286.

38. Steegmaier, M., Hoffmann, M., Baum, A., Lenart, P., Petronczki, M., Krssak, M., et al. (2007). BI 2536, a potent and selective inhibitor of polo-like kinase 1, inhibits tumor growth in vivo. Current Biology, 17(4), 316-322.

39. Lenart, P., Petronczki, M., Steegmaier, M., Di Fiore, B., Lipp, J. J., Hoffmann, M., et al. (2007). The small-molecule inhibitor BI 2536 reveals novel insights into mitotic roles of polo-like kinase 1 . Current Biology, 17(4), 304-315.

40. Peters, U., Cherian, J., Kim, J. H., Kwok, B. H., \& Kapoor, T. M. (2006). Probing cell-division phenotype space and Polo-like kinase function using small molecules. Nat Chem Biol, 2(11), 618-626.

41. Santamaria, A., Neef, R., Eberspacher, U., Eis, K., Husemann, M., Mumberg, D., et al. (2007). Use of the Novel Plk1 Inhibitor ZKThiazolidinone to Elucidate Functions of Plk1 in Early and Late Stages of Mitosis. Molecular biology of the cell.

42. Ditchfield, C., Johnson, V. L., Tighe, A., Ellston, R., Haworth, C., Johnson, T., et al. (2003). Aurora B couples chromosome alignment with anaphase by targeting BubR1, Mad2, and CenpE to kinetochores. Journal of Cell Biology, 161(2), 267-280.

43. Hauf, S., Cole, R. W., LaTerra, S., Zimmer, C., Schnapp, G., Walter, R., et al. (2003). The small molecule Hesperadin reveals a role for Aurora B in correcting kinetochore-microtubule attachment and in maintaining the spindle assembly checkpoint. Journal of Cell Biology, 161(2), 281-294. 
44. Lipp, J. J., Hirota, T., Poser, I., \& Peters, J. M. (2007). Aurora B controls the association of condensin I but not condensin II with mitotic chromosomes. Journal of Cell Science, $120(\mathrm{Pt} 7), 1245$ 1255.

45. Harrington, E. A., Bebbington, D., Moore, J., Rasmussen, R. K., Ajose-Adeogun, A. O., Nakayama, T., et al. (2004). VX-680, a potent and selective small-molecule inhibitor of the Aurora kinases, suppresses tumor growth in vivo. Natural Medicines, 10(3), 262-267.

46. Carpinelli, P., Ceruti, R., Giorgini, M. L., Cappella, P., Gianellini, L., Croci, V., et al. (2007). PHA-739358, a potent inhibitor of Aurora kinases with a selective target inhibition profile relevant to cancer. Mol Cancer Ther, 6(12 Pt 1), 3158-3168.
47. Wilkinson, R. W., Odedra, R., Heaton, S. P., Wedge, S. R., Keen, N. J., Crafter, C., et al. (2007). AZD1152, a selective inhibitor of Aurora B kinase, inhibits human tumor xenograft growth by inducing apoptosis. Clinical cancer research, 13(12), 36823688 .

48. Turner, N. C., Lord, C. J., Iorns, E., Brough, R., Swift, S., Elliott, R., et al. (2008). A synthetic lethal siRNA screen identifying genes mediating sensitivity to a PARP inhibitor. European Molecular Biology Organization journal, 27(9), 13681377.

49. Musacchio, A., \& Salmon, E. D. (2007). The spindle-assembly checkpoint in space and time. Nature reviews. Molecular cell biology, 8(5), 379-393. 